\title{
MODEL OF NONLINEAR OPERATION OF A WAVEGUIDE LASER WITH GAUSSIAN OUTPUT MIRROR
}

\author{
P. WITOŃSKI ${ }^{a *}$ AND P. SzCZEPAŃSKI ${ }^{a, b, c}$ \\ ${ }^{a}$ Institute of Microelectronics and Optoelectronics, Warsaw University of Technology \\ Koszykowa 75, 00-662 Warsaw, Poland \\ ${ }^{b}$ Institute of Telecommunication, Szachowa 1, 04-894 Warsaw, Poland \\ ${ }^{c}$ Institute of Electronic Materials Technology \\ Wólczyńska $133,01-919$ rsaw, Poland
}

We present an approximate analysis of the nonlinear operation of the hollow-waveguide laser with Gaussian reflectivity profile output mirror, including gain saturation and longitudinal- as well as transverse-field distribution of the laser mode. The model presented is general and can be applied to the study of an arbitrary configuration of the waveguide laser. In particular, the laser characteristics show the influence of the position of the output mirror and the Gaussian mirror parameter on the power efficiency of the laser system. It was shown that optimal position of the output mirror, which provides maximal power efficiency (with other parameters constant), depends on output power level and the mirror reflectivity coefficient.

PACS numbers: 42.60.Da, 42.60.Lh

\section{Introduction}

Hollow-waveguide lasers [1-4] have become one of the most commonly used laser structures in many applications. They have been intensively studied theoretically [5-11] as well as experimentally [12-19]. The efforts to demonstrate and characterise different devices with different gases (especially with $\mathrm{CO}_{2}$ ) as an active medium, and experiments with different resonators and waveguide types have been reviewed in several very good papers [20-22]. However, most of the theoretical works consider mode behaviour of the passive resonators of the various configurations. In particular, mode structure and loss minimisation in waveguide resonators have been analysed. It has been found that three low-loss reflectors geometries exist, i.e., when the large radius $R$ mirrors are very near the guide (so called "dual case I"), when the distance between the large radius $R$ mirror and the guide entrance is approximately equal to $R$ ("dual case II"), and finally, when the

*e-mail: p.witonski@imio.pw.edu.pl 
mirrors are set at the Rayleigh range of the $\mathrm{EH}_{11}$ mode approximating Gaussian mode (i.e., TEM $_{00}$ beam having maximum overlap with $\mathrm{EH}_{11}$ across the guide aperture - "dual case III", see for example [23]).

Moreover, transverse modes of active hollow waveguide resonators have been investigated [11]. It has been shown that the deviations from passive waveguide modes, resulting purely from the presence of the active medium, can be neglected for parameters representing typical experimental situations of single-guide $\mathrm{CO}_{2}$ waveguide lasers (with a guide cross-section of a few $\mathrm{mm}^{2}$ ). The thermally-induced index profile of laser gas already causes more efficient inversion exploitation over the cross-section and supports transversal, single mode operation of the laser.

More recently, the approximate method describing nonlinear operation of the hollow-waveguide laser including transverse field distribution has been developed [24-27]. A simple approximate expression describing dependence of the output power on the global small-signal gain coefficient, the distributed losses, the mirror reflectance, and the transverse mode distribution has been derived. However, the model presented in [24] is confined to the "dual case I" and it gives the results, which are in good agreement with the exact solutions [28-37] in the low-power limit.

More general model of the nonlinear operation of the hollow-waveguide, valid for the arbitrary configuration of the waveguide laser and the wider power range, has been presented in [38]. It has been shown that the optimal position of the output mirror (which provides maximal power efficiency of the laser system with the other parameters constant) depends on the output power level and the mirror reflectivity coefficient. Moreover, the laser characteristics show that introducing an additional device into the cavity causes dependence of the power efficiency on which end of the laser the light power is extracted from.

In this paper we extend the approximate model presented in [38] to describe the nonlinear operation of the hollow-waveguide laser with Gaussian mirror [39-48]. In general, the utilization of the Gaussian-reflectivity mirror provides the good transverse selectivity for the lowest-order (fundamental) mode. Thus, using Gaussian reflectivity mirror as a transmission one makes possible improving quality of the generated beam. However, because the Gaussian behaves in the different way than classical mirror [49], the boundary conditions for the electric field of the laser mode requires some modifications (in comparison with the model presented in [38]). Moreover, we can also expect that the coupling losses between waveguides modes and free-space modes will be changed and different low-loss reflector geometries will exist.

In the next section, the theoretical model of the nonlinear operation of the hollow-waveguide laser with Gaussian mirror is presented. With the help of the energy theorem and threshold field approximation an approximate expression of the small signal gain as a function of output power and the characteristic system parameters is derived. In particular, this expression makes possible the investigation of the effect of the Gaussian mirror on nonlinear operation of the hollow-waveguide laser. It is worth noting that it is valid for arbitrary laser configuration. Laser characteristics showing the behaviour of the coupling losses and power efficiency of the laser structure are presented in Sec. 3. Section 4 gives the conclusions. 


\section{Energy theorem for the hollow-waveguide lasers}

We analyse the rectangular waveguide laser shown in Fig. 1. The laser configuration consists of hollow waveguide with distributed losses $\alpha_{0}$ (in general, different for different transverse modes) and the length $L$, filled with the gas active medium with a small-signal gain coefficient $g_{0}$. The end mirrors "classical" and "Gaussian", are set at distance $z_{1}$ and $z_{2}$ from the waveguide, respectively. The "classical" mirror has curvature radius $R_{\mathrm{c}}$. Its amplitude reflectivity coefficient $\rho_{\mathrm{c}}$ is uniform in the plane of the mirror. The Gaussian has curvature radius $R_{\mathrm{g}}$. Its amplitude reflectivity coefficient $\rho_{\mathrm{g}}$ varies with the radial distance from the center of the mirror according to $\rho_{\mathrm{g}}(r)=\rho_{0} \exp \left(-r^{2} / w_{\mathrm{g}}^{2}\right)$, where $w_{\mathrm{g}}$ is the Gaussian mirror parameter $[39,40]$ and $\rho_{0}$ is the amplitude reflectivity at the centre of the mirror.

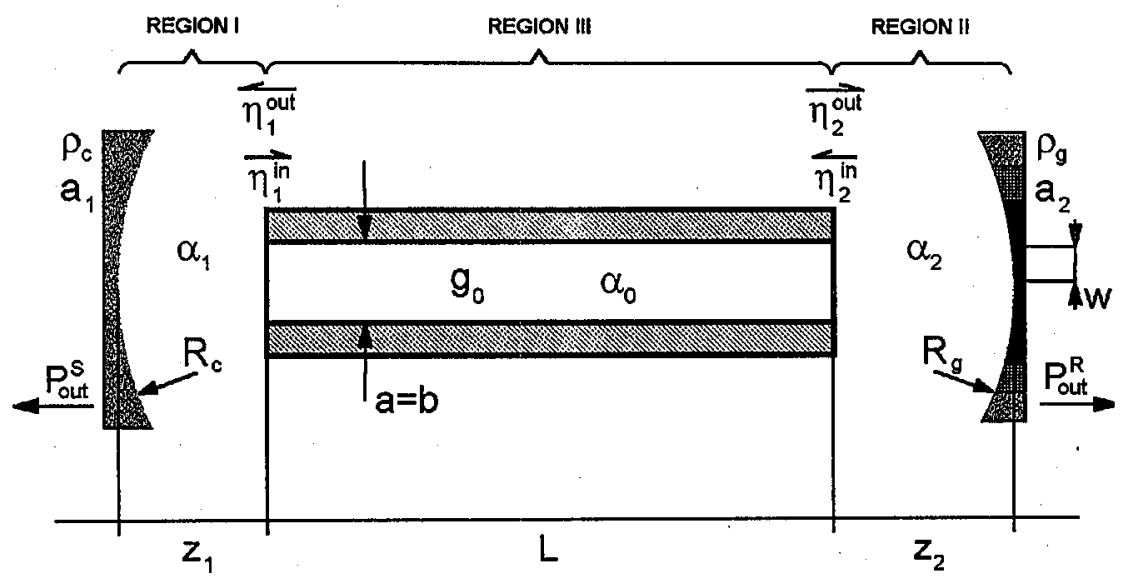

Fig. 1. Hollow-waveguide laser configuration considered in this paper.

For generality we also assume nonzero distributed losses in regions between the waveguide and the end mirrors (regions I and II in Fig. 1), described by the distributed loss coefficients $\alpha_{1}$ and $\alpha_{2}$, respectively. These distributed losses can represent many effects, in particular, they can represent losses in other extracavity' devices such as modulators and polarizers.

For our laser structure the electric field of the laser mode can be written (similarly as in [38]) in the following form:

$$
\begin{aligned}
& E_{R, q n m}^{\mathrm{I}, \mathrm{II}, \mathrm{III}}(x, y, z)=R_{q n m}^{\mathrm{I}, \mathrm{II}, \mathrm{III}}(z) E_{R, q n m}^{\mathrm{I}, \mathrm{II}, \mathrm{III}}(x, y, z), \\
& E_{S, q n m}^{\mathrm{I}, \mathrm{II}, \mathrm{III}}(x, y, z)=S_{q n m}^{\mathrm{I}, \mathrm{II}, \mathrm{III}}(z) E_{S, q n m}^{\mathrm{I}, \mathrm{II}, \mathrm{III}}(x, y, z),
\end{aligned}
$$

where $R_{q n m}^{\mathrm{I}, \mathrm{II}, \mathrm{III}}(z)$ and $S_{q n m}^{\mathrm{I}, \mathrm{II}, \mathrm{III}}(z)$ are the complex amplitudes of the two counter-running waves of the laser mode, in free-space (regions I and II) and in the waveguide (region III), respectively. $E_{R, q n m}^{\mathrm{I}, \mathrm{II}, \mathrm{III}}(x, y, z)$ and $E_{S, q n m}^{\mathrm{I}, \mathrm{II}, \mathrm{III}}(x, y, z)$ describe appropriate transverse field distribution in the free-space (the Hermite-Gauss or the Laguerre-Gauss modes [27]). In our case in region I we have $E_{R, q n m}^{\mathrm{I}}(x, y, z)=$ $E_{S, q n m}^{\mathrm{I}}(x, y, z)$. In region II, because of Gaussian mirror acting, we have 
$E_{R, q n m}^{\mathrm{II}}(x, y, z) \neq E_{S, q n m}^{\mathrm{II}}(x, y, z)$. In the waveguide we have the waveguide modes $[5,12] E_{R, q n m}^{\mathrm{III}}(x, y, z)=E_{S, q n m}^{\mathrm{III}}(x, y, z)=E_{q n m}^{\mathrm{III}}(x, y, z)$. Subscript $q$ denotes longitudinal mode number. Subscript $n m$ describes transverse mode number. In our approach we assume that the active medium is homogeneously broadened, spatial hole burning and the mode competition are neglected, and finally, the frequency of the laser mode is centrally tuned.

For this assumption, an energy relation for our laser structure, according to [38], can be written in the following form:

$$
\begin{aligned}
& \frac{\mathrm{d}}{\mathrm{d} z}\left(\left|R_{q n m}^{\mathrm{I}, \mathrm{II}, \mathrm{III}}(z)\right|^{2}-\left|S_{q n m}^{\mathrm{I}, \mathrm{II}, \mathrm{III}}(z)\right|^{2}\right)=-\left.2 \alpha_{1}\left(\left|R_{q n m}^{\mathrm{I}}\right|^{2}+\left|S_{q n m}^{\mathrm{I}}\right|^{2}\right)\right|_{0} ^{z_{1}} \\
& -\left.2 \alpha_{2}\left(\left|R_{q n m}^{\mathrm{II}}\right|^{2}+\left|S_{q n m}^{\mathrm{II}}\right|^{2}\right)\right|_{z_{1}+L} ^{z_{1}+L+z_{2}}-\left.2 \alpha_{0}\left(\left|R_{q n m}^{\mathrm{III}}\right|^{2}+\left|S_{q n m}^{\mathrm{III}}\right|^{2}\right)\right|_{z_{1}} ^{z_{1}+L} \\
& +\left.\frac{k_{0}}{\beta_{m n}\left\|\mid E_{q n m}^{\mathrm{III}}\right\|} \iint_{x, y} \frac{g_{0} f(x, y, z)\left(\left|R_{q n m}^{\mathrm{III}}\right|^{2}+\left|S_{q n m}^{\mathrm{III}}\right|^{2}\right)\left|E_{q n m}^{\mathrm{III}}\right|^{2}}{1+\frac{\left(\left|R_{q n m}^{\mathrm{III}}\right|^{2}+\left|S_{q n m}^{\operatorname{III}}\right|^{2}\right)\left|E_{q n m}^{\operatorname{III}}\right|^{2}}{P_{\mathrm{S}}}} \mathrm{d} x \mathrm{~d} y\right|_{z_{1}} ^{z_{1}+L}
\end{aligned}
$$

where $P_{\mathrm{S}}$ is the saturation power and the normalisation factor $\left\|E_{\text {qnm }}^{\mathrm{III}}\right\|$ is given by $\left\|E_{q n m}^{\mathrm{III}}\right\|=\iint\left|E_{q n m}^{\mathrm{III}}\right|^{2} \mathrm{~d} x \mathrm{~d} y$, where the integral is carried out over the cross-section. of the waveguide. Moreover, the normalised function $f(x, y, z)$ describes the spatial distribution of the small signal gain, which in general, depends on the pumping of the active medium.

In order to integrate Eq. (2.2) over the length of the structure we should specify the boundary conditions. Because of the Gaussian mirror, they should be modified in comparison to the model presented in [38]. Thus, in our case the new boundary conditions can be written in the following form. At the ends of laser, $z=0$ and $z=L_{\text {tot }}$, we have

$$
\begin{aligned}
& \left|R_{q n m}^{\mathrm{I}}(0)\right|^{2}=\rho_{\mathrm{c}}{ }^{2}\left|S_{q n m}^{\mathrm{I}}(0)\right|^{2}, \\
& \left(1-a_{1}^{2}-\rho_{\mathrm{c}}{ }^{2}\right)\left|S_{q n m}^{\mathrm{I}}(0)\right|^{2} \iint_{x, y}\left|E_{q n m}^{\mathrm{I}}(x, y, 0)\right|^{2} \mathrm{~d} x \mathrm{~d} y=P_{\text {out }}^{S},
\end{aligned}
$$

and

$$
\begin{aligned}
& \left|R_{q n m}^{\mathrm{II}}\left(L_{\mathrm{tot}}\right)\right|^{2} \rho_{\mathrm{eff}}^{2}=\left|S_{q n m}^{\mathrm{II}}\left(L_{\mathrm{tot}}\right)\right|^{2}, \\
& \left(1-a_{2}^{2}-\rho_{\mathrm{eff}}^{2}\right)\left|R_{q n m}^{\mathrm{II}}\left(L_{\mathrm{tot}}\right)\right|^{2} \iint_{x, y}\left|E_{q n \dot{m}}^{\mathrm{II}}\left(x, y, L_{\mathrm{tot}}\right)\right|^{2} \mathrm{~d} x \mathrm{~d} y=P_{\text {out }}^{R},
\end{aligned}
$$

where $a_{1}$ and $a_{2}$ are the point loss coefficients at the mirrors, total resonator length equals to $L_{\text {tot }}=z_{1}+L+z_{2}, P_{\text {out }}=P_{\text {out }}^{R}+P_{\text {out }}^{S}$ describes the total power escaping from the laser and the effective reflectivity of the Gaussian mirror $\rho_{\mathrm{eff}}$, which, in general can be defined as [46]

$$
\rho_{\mathrm{eff}}=\rho_{0}\left\{\frac{\iint_{x, y}\left|E_{R, q n m}^{\mathrm{II}}\left(x, y, L_{\mathrm{tot}}\right) \exp \left(-\frac{x^{2}+y^{2}}{w_{\mathrm{g}}^{2}}\right)\right|^{2} \mathrm{~d} x \mathrm{~d} y}{\iint_{x, y}\left|E_{R, q n m}^{\mathrm{II}}\left(x, y, L_{\mathrm{tot}}\right)\right|^{2} \mathrm{~d} x \mathrm{~d} y}\right\}^{\frac{1}{2}}
$$


The boundary conditions at the waveguides ends, $z=z_{1}$ and $z=z_{1}+L$, can be written as follows:

$$
\left|R_{q n m}^{\mathrm{I}}\left(z_{1}\right)\right|^{2} \eta_{1}^{\mathrm{in}}=\left|R_{q n m}^{\mathrm{III}}\left(z_{1}\right)\right|^{2},\left|R_{q n m}^{\mathrm{III}}\left(z_{1}+L\right)\right|^{2} \eta_{2}^{\text {out }}=\left|R_{q n m}^{\mathrm{II}}\left(z_{1}+L\right)\right|^{2},
$$

and

$$
\left|S_{q n m}^{\mathrm{III}}\left(z_{1}\right)\right|^{2} \eta_{1}^{\text {out }}=\left|S_{q n m}^{\mathrm{I}}\left(z_{1}\right)\right|^{2},\left|S_{q n m}^{\mathrm{II}}\left(z_{1}+L\right)\right|^{2} \eta_{2}^{\text {in }}=\left|S_{q n m}^{\mathrm{III}}\left(z_{1}+L\right)\right|^{2} .
$$

$\eta_{1(2)}^{\text {out }}$ is the power coupling coefficient between the waveguide modes and free-space modes [38] defined as follows:

$$
\begin{gathered}
\eta_{1(2)}^{\text {out }}= \\
\frac{\left(\iint_{x, y}\left\{E_{q n m}^{\mathrm{III}}\left[x, y, z_{1}\left(z_{1}+L\right)\right] E_{S(R) q n m}^{\mathrm{IIII})}\left[x, y, z_{1}\left(z_{1}+L\right)\right]^{*}\right\} \mathrm{d} x \mathrm{~d} y\right)^{2}}{\iint_{x, y}\left|E_{q n m}^{\mathrm{III}}\left[x, y, z_{1}\left(z_{1}+L\right)\right]\right|^{2} \mathrm{~d} x \mathrm{~d} y \iint_{x, y}\left|E_{S(R) g n m}^{\mathrm{I}(\mathrm{II})}\left[x, y, z_{1}\left(z_{1}+L\right)\right]\right|^{2} \mathrm{~d} x \mathrm{~d} y},
\end{gathered}
$$

where $E_{S(R) q n m}^{\mathrm{I}(\mathrm{II})}$ is free-space mode excited by waveguide mode. $\eta_{1(2)}^{\text {in }}$ is the power coupling coefficient between free-space modes and the waveguide modes [38] defined as follows:

$$
\begin{gathered}
\eta_{1(2)}^{\mathrm{in}}= \\
\frac{\left(\iint_{x, y}\left[E_{q n m}^{\mathrm{III}}\left[x, y, z_{1}\left(z_{1}+L\right)\right] E_{R(S) g n m}^{\mathrm{I}(\mathrm{II})}\left[x, y, z_{1}\left(z_{1}+L\right)\right]^{*}\right] \mathrm{d} x \mathrm{~d} y\right)^{2}}{\iint_{x, y}\left|E_{q n m}^{\mathrm{III}}\left[x, y, z_{1}\left(z_{1}+L\right)\right]\right|^{2} \mathrm{~d} x \mathrm{~d} y \iint_{x, y}\left|E_{R(S) q n m}^{\mathrm{I}(\mathrm{II})}\left[x, y, z_{1}\left(z_{1}+L\right)\right]\right|^{2} \mathrm{~d} x \mathrm{~d} y}
\end{gathered}
$$

where $E_{R(S) q n m}^{\mathrm{I}(\mathrm{II})}$ is free space mode incident on waveguide cross-section. It is worth noting that in general, for the arbitrary waveguide structure geometry with arbitrary end mirrors, $\eta_{1(2)}^{\text {in }}$ and $\eta_{1(2)}^{\text {out }}$ can be different.

In order to calculate effective reflectivity of the Gaussian mirror and power. coupling coefficients we need to specify appropriate transverse field distribution in three regions of our laser structure.

In general, the transverse distribution of the laser mode inside the waveguide is determined by the geometry of the waveguide and is described by waveguide modes $[5,12,38]$

$$
E_{q n m}^{\operatorname{III}}(x, y, z)=\left\{\begin{array}{l}
\cos \\
\sin
\end{array}\left(\frac{n \pi x}{2 a}\right) \times \begin{array}{l}
\cos \\
\sin
\end{array}\left(\frac{m \pi y}{2 b}\right)\right\}, \quad n, m=\left\{\begin{array}{c}
\text { even } \\
\text { odd }
\end{array}\right.
$$

In our model, because of the presence of the Gaussian mirror, we assume that laser action inside the waveguide develops on the fundamental waveguide mode $\mathrm{EH}_{11}$ given by Eq. (2.9) with $n=m=1$.

On the other hand, in free-space region the electric field is described by the Hermite-Gauss modes. However, because of the transverse mode selectivity of the Gaussian mirror, we can assume that in this region, the laser operates on fundamental Gaussian mode 


$$
\begin{aligned}
& E_{R(S) q n m}^{\mathrm{I}(\mathrm{II})}(x, y, z)=\frac{w_{0}}{w_{\mathrm{g}}(z)} \exp \left\{-\frac{\mathrm{i} k\left(x^{2}+y^{2}\right)}{2}\left(\frac{1}{R(z)}-\frac{\mathrm{i} \lambda}{\pi w_{\mathrm{g}}^{2}(z)}\right)\right\} \\
& =\frac{w_{0}}{w_{\mathrm{g}}(z)} \exp \left\{-\frac{\mathrm{i} k\left(x^{2}+y^{2}\right)}{2} \frac{1}{q(z)}\right\},
\end{aligned}
$$

where $w_{0}$ is the beam waist, $w_{\mathrm{g}}$ is the Gaussian parameter, the phase front curvature radius is denoted by $R, \lambda$ is the wavelength in the free-space region, $k=\frac{2 \pi}{\lambda}$. is the wavenumber and $q$ is the complex beam parameter.

Now, using the field distributions, Eq. (2.9) with $n=m=1$ and Eq. (2.10), it is possible to calculate spatial overlap integrals between waveguide and free-space describing coupling efficiency, Eqs. (2.7) and (2.8), as well as effective reflectivity $\rho_{\text {eff }}$ of the Gaussian mirror, Eq. (2.5).

First, we consider the coupling between the waveguide mode and free-space mode. It is well known [5] that the fundamental waveguide mode excites with the maximal efficiency the fundamental Gaussian mode having Gaussian parameter equal to $w_{\mathrm{g}}=0.703 a$ (where $a$ is the waveguide cross-section) and flat phase front, i.e., $1 / R=0$ (see also [49]). In this case the coupling efficiency $\eta_{1(2)}^{\text {out }}$, which is simultaneously power transmission from waveguide to free-space, is equal to 0.98 . Thus, we can assume that the waveguide mode leaving the waveguide transforms itself into the fundamental (free-space) Gaussian mode having beam parameters equal to $w_{\mathrm{g}}=0.703 a$ and $R=\infty$. Now, we can use Kogelnik transformation [42] to determine the spatial field distribution of the laser mode in free space (i.e. in regions $I$ and II), instead of time consuming analysis based on diffraction theory. Thus, using appropriate $A B C D$ matrices (one including reflection from the classical mirror and the second one taking into account reflection from the Gaussian mirror $[39,40])$ it is possible to calculate the transverse field distribution of the wave incident on the Gaussian mirror and waves coming back to the waveguide, i.e., $E_{R, q n m}^{\mathrm{I}}\left(x, y, z_{1}\right)$ and $E_{S^{\prime}, q n m}^{\mathrm{II}}\left(x, y, L+z_{1}\right)$, respectively. Furthermore, using Eq. (2.8), the power coupling coefficients $\eta_{1(2)}^{\text {int }}$ between the free-space and the waveguide modes as well as effective reflectivity of the Gaussian mirror $\rho_{\text {eff }}$ defined by Eq. (2.5) can be determined.

Integration of Eq. (2.2), taking into account boundary conditions Eqs. (2.3), (2.4), (2.6) results in

$$
\begin{aligned}
& \left|R_{q n m}^{\mathrm{II}}\left(L_{\mathrm{tot}}\right)\right|^{2}\left(1-\rho_{\mathrm{eff}}^{2}\right)+\left|S_{q n m}^{\mathrm{I}}(0)\right|^{2}\left(1-\rho_{\mathrm{c}}{ }^{2}\right)+\left|R_{q n m}^{\mathrm{I}}\left(z_{1}\right)\right|^{2}\left(1-\eta_{1}^{\mathrm{in}}\right) \\
& +\left|S_{q n m}^{\mathrm{III}}\left(z_{1}\right)\right|^{2}\left(1-\eta_{1}^{\mathrm{out}}\right)+\left|R_{q n m}^{\mathrm{III}}\left(z_{1}+L\right)\right|^{2}\left(1-\eta_{2}^{\mathrm{out}}\right) \\
& +\left|S_{q n m}^{\mathrm{II}}\left(z_{1}+L\right)\right|^{2}\left(1-\eta_{2}^{\mathrm{in}}\right)+\int_{0}^{z_{1}} 2 \alpha_{1}\left(\left|\mathrm{R}_{\mathrm{qnm}}^{\mathrm{I}}(\mathrm{z})\right|^{2}+\left|\mathrm{S}_{\mathrm{qnm}}^{\mathrm{I}}(\mathrm{z})\right|^{2}\right) \mathrm{dz} \\
& +\int_{z_{1}}^{z_{1}+L} 2 \alpha_{0}\left(\left|R_{q n m}^{\mathrm{III}}(z)\right|^{2}+\left|S_{q n m}^{\mathrm{III}}(z)\right|^{2}\right) \mathrm{d} z
\end{aligned}
$$




$$
\begin{aligned}
& +\int_{z_{1}+L}^{L_{\mathrm{tot}}} 2 \alpha_{2}\left(\left|R_{q n m_{m n}}^{\mathrm{II}}(z)\right|^{2}+\left|S_{q n m_{m n}}^{\mathrm{II}}(z)\right|^{2}\right) \mathrm{d} z=\frac{k}{\beta_{m n}|| E_{q n m}^{\mathrm{III}} \|} \\
& \times \int_{z_{1}}^{z_{1}+L} \mathrm{~d} z \iint_{x, y} \frac{g_{0} f(x, y, z)\left(\left|R_{q n m}^{\mathrm{III}}(z)\right|^{2}+\left|S_{q n m}^{\mathrm{III}}(z)\right|^{2}\right)\left|E_{q n m}^{\mathrm{III}}\right|^{2}}{1+\frac{\left(\left|R_{q n m}^{\mathrm{III}}(z)\right|^{2}+\left|S_{q n m}^{\mathrm{III}}(z)\right|^{2}\right)\left|E_{q n m}^{\mathrm{III}}\right|^{2}}{P_{\mathrm{S}}}} \mathrm{d} x \mathrm{~d} y .
\end{aligned}
$$

This relation is exact and describes the energy conservation theorem for hollow-waveguide lasers having resonator with the Gaussian mirror.

Similarly as in [26], we use that relation as a starting point in our approximate analysis. We approximate the field distribution appearing in the energy theorem, Eq. (2.11), including nonlinearities, by the one existing in the linear structure, i.e. satisfying linear equations (valid at the threshold operation) for the boundary conditions, Eqs. (2.3), (2.4), (2.6). It is worth noting that approximation has been verified for two-mirror lasers [50] as well as for distributed feedback lasers [26]. Moreover, this assumption has been also confirmed experimentally [11]: According to this, we assume that $R_{q n m}^{\mathrm{I}, \mathrm{II}, \mathrm{III}}(z)$ and $S_{q n m}^{\mathrm{I}, \mathrm{II}, \mathrm{III}}(z)$ are proportional to the threshold field distribution. Thus, we have in the region $\mathrm{I}$ (for $0 \leq z \leq z_{1}$ )

$$
\begin{aligned}
& \left|R_{q n m}^{\mathrm{I}}(z)\right|^{2}=A^{2} \exp \left(-2 \alpha_{1} z\right), \\
& \left|S_{q n m}^{\mathrm{I}}(z)\right|^{2}=\frac{A^{2}}{\rho_{\mathrm{c}}^{2}} \exp \left(2 \alpha_{1} z\right),
\end{aligned}
$$

in the region II (for $L+z_{1} \leq z \leq L_{\mathrm{tot}}$ )

$$
\begin{aligned}
& \left|R_{q n m}^{\mathrm{II}}(z)\right|^{2}=\frac{C^{2}}{\rho_{\mathrm{eff}}^{2}} \exp \left[-2 \alpha_{2}\left(z-L_{\mathrm{tot}}\right)\right], \\
& \left|S_{q n m}^{\mathrm{II}}(z)\right|^{2}=C^{2} \exp \left[2 \alpha_{2}\left(z-L_{\mathrm{tot}}\right)\right],
\end{aligned}
$$

and in the waveguide (region III, $z_{1} \leq z \leq L+z_{1}$ )

$$
\begin{aligned}
\left|R_{q n m}^{\mathrm{III}}(z)\right|^{2} & =B^{2} \exp \left[2 \gamma\left(z-z_{1}\right)\right], \\
\left|S_{q n m}^{\mathrm{III}}(z)\right|^{2} & =\frac{B^{2}}{\rho_{\mathrm{c}}^{2} \eta_{1}^{\text {in }} \eta_{1}^{\text {out }} \exp \left(-4 \alpha_{1} z_{1}\right)} \exp \left[-2 \gamma\left(z-z_{1}\right)\right],
\end{aligned}
$$

where constant $\gamma$ is given by

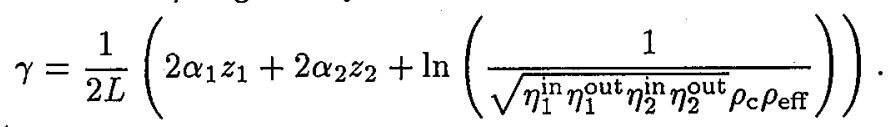

According to the boundary conditions (2.3), (2.4) and (2.6), the relations between the field amplitudes $A, B$, and $C$, can be written in the following form:

$$
A^{2}=B^{2} \frac{\exp \left(2 \alpha_{1} z_{1}\right)}{\eta_{1}^{\text {in }}} \text { and } C^{2}=B^{2} \exp \left(2 \alpha_{1} z_{1}\right) \frac{\rho_{\text {eff }}}{\rho_{\mathrm{c}}} \sqrt{\frac{\eta_{2}^{\text {out }}}{\eta_{1}^{\text {in }} \eta_{1}^{\text {out }} \eta_{2}^{\text {in }}}}
$$

Moreover, taking into account boundary conditions (2.3), (2.4) and (2.6), the field distributions (2.9), (2.10) and the relations (2.16), we can relate the field amplitude $B$ to the output power in the following way: 


$$
\begin{aligned}
B^{2}= & P_{\text {out }} \frac{\rho_{\mathrm{c}}}{\exp \left(2 \alpha_{1} z_{1}\right)}\left(\frac{1-a_{1}^{2}-\rho_{\mathrm{c}}^{2}}{\rho_{\mathrm{c}} \eta_{1}^{\text {in }}}\left\|E_{S, q m n}^{\mathrm{I}}(x, y, 0)\right\|\right. \\
& \left.+\frac{1-a_{2}^{2}-\rho_{\mathrm{eff}}^{2}}{\rho_{\mathrm{eff}}} \sqrt{\frac{\eta_{2}^{\text {out }}}{\eta_{1}^{\text {in }} \eta_{1}^{\text {out }} \eta_{2}^{\text {in }}}}\left\|E_{R, q m n}^{\mathrm{II}}\left(x, y, L_{\mathrm{tot}}\right)\right\|\right)^{-1} .
\end{aligned}
$$

Combining the approximate field distribution for $R_{m n}^{q}(z)$ and $S_{m n}^{q}(z)$, Eqs. (2.12)(2.14), and the expression for the field amplitude, Eq. (2.17) (together with the relations (2.16)), with the energy theorem (2.11) we obtain

$$
\begin{aligned}
g_{0}= & \left\{\left[(\exp (2 \gamma L)-1)-(\exp (-2 \gamma L)-1) \frac{\exp \left(4 \alpha_{1} z_{1}\right)}{\rho_{\mathrm{c}}^{2} \eta_{1}^{\text {in }} \eta_{1}^{\text {out }}}\right]\left(1+\frac{\alpha_{0}}{\gamma}\right)\right\} \\
& \times\left(\frac{k_{0}}{\beta_{m n}\left|E_{q m n}^{\text {III }}\right|} \int_{z_{1}}^{z_{1}+L} \mathrm{~d} z \iint_{x, y} \frac{f(x, y, z) \boldsymbol{D}(z)\left|E_{q m n}^{\mathrm{III}}\right|^{2}}{1+\frac{P_{\text {out }}}{P_{\mathrm{S}}} \frac{\left.\boldsymbol{D}_{(z) \mid E_{m m n}}\right|^{2}}{N}} \mathrm{~d} x \mathrm{~d} y\right)^{-1},
\end{aligned}
$$

where the longitudinal intensity distribution is given by

$$
\boldsymbol{D}(z)=\exp \left[2 \gamma\left(z-z_{1}\right)\right]+\exp \left[-2 \gamma\left(z-z_{1}\right)\right] \frac{\exp \left(4 \alpha_{1} z_{1}\right)}{\rho_{\mathrm{c}}^{2} \eta_{1}^{\text {in }} \eta_{1}^{\text {out }}},
$$

and the normalisation constant $N$ is defined by

$$
\begin{aligned}
N= & \frac{\exp \left(2 \alpha_{1} z_{1}\right)}{\rho_{\mathrm{c}}}\left[\frac{1-a_{1}^{2}-\rho_{\mathrm{c}}^{2}}{\rho_{\mathrm{c}} \eta_{1}^{\text {in }}}\left\|E_{S, q m n}^{\mathrm{I}}(x, y, 0)\right\|\right. \\
& \left.+\frac{1-a_{2}^{2}-\rho_{\mathrm{eff}}^{2}}{\rho_{\mathrm{eff}}} \sqrt{\frac{\eta_{2}^{\text {out }}}{\eta_{1}^{\text {in }} \eta_{1}^{\text {out }} \eta_{2}^{\text {in }}}}\left\|E_{R, q m n}^{\mathrm{II}}\left(x, y, L_{\mathrm{tot}}\right)\right\|\right] .
\end{aligned}
$$

Relation (2.18) is an approximate expression relating the small signal gain coefficient to the normalised output power and system parameters for hollow-waveguide lasers having Gaussian mirror. This relation is valid for arbitrary configuration of the cavity.

Using this formula the systematic study of nonlinear single mode operation of the waveguide laser with Gaussian mirror is performed. The output power characteristics revealing an influence of system parameters on laser operation such as arbitrary mirror reflectivity, Gaussian mirror parameter and geometry of the cavity are obtained.

\section{Laser characteristics}

In Sec. 3 we describe the results of numerical evaluation for the rectangular waveguide structure shown in Fig. 1. In our calculations we assume uniform pumping of the active medium in waveguide region $(f(x, y, z) \equiv 1)$. The power. is extracted through the Gaussian mirror and the reflectivity of the conventional mirror is equal to unity. The radius of the curvature of the end mirrors is equal to $R_{\mathrm{c}}=R_{\mathrm{g}}=200 \mathrm{~cm}$, the length of the waveguide is equal to $L=1 \mathrm{~m}$. In our calculations, the spatial distribution of the waveguide mode is described by 
the passive waveguide modes, since, according to [38], the deviations from passive waveguide modes resulting purely from the presence of the active medium, can be neglected for typical experimental parameters of single-guide $\mathrm{CO}_{2}$ waveguide laser considered in this paper.

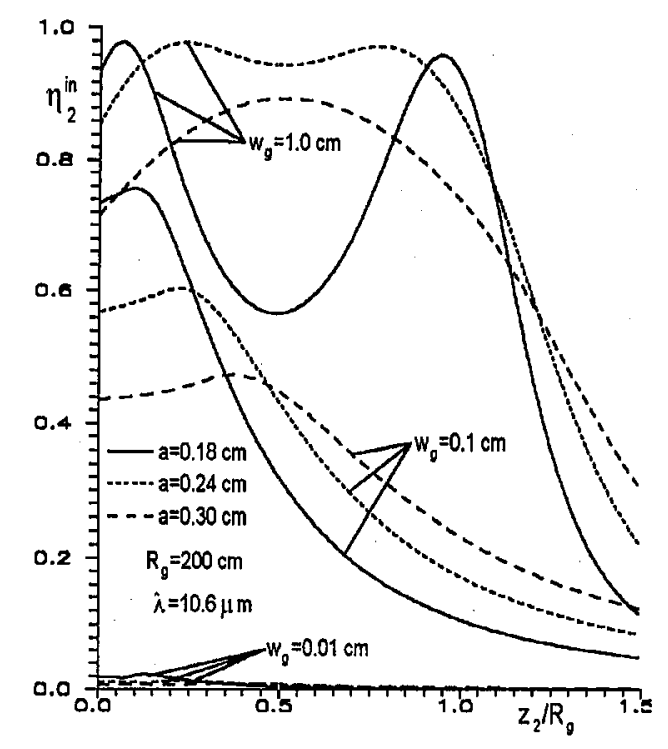

Fig. 2. Power coupling coefficient $\eta_{2}^{\text {in }}$ as a function of the normalised to the mirror curvature radius Gaussian mirror position $z_{2} / R_{\mathrm{g}}$ for three values of the Gaussian mirror parameter $w_{\mathrm{g}}$. Gaussian mirror curvature radius is equal to $R_{\mathrm{g}}=200 \mathrm{~cm}$.

First we consider the behaviour of the power coupling coefficient $\eta_{2}^{\text {in }}$ between the free-space wave reflected from the Gaussian mirror (region II) and incident on the waveguide aperture (at $z=z_{1}+L$; see Fig. 1) and the waveguide mode. In Fig. 2 this power coupling coefficient, i.e. $\eta_{2}^{\text {in }}$, is plotted as a function of the normalised to the mirror curvature radius Gaussian mirror position. We assume the square cross-section of the waveguide and consider height (or width) of the waveguide as a parameter. The characteristics are obtained for two values of the Gaussian mirror parameter $w_{\mathrm{g}}=1 \mathrm{~cm}$ and $w_{\mathrm{g}}=0.1 \mathrm{~cm}$. It is worth noting that the Gaussian mirror tends to the classical one when the value of the parameter $w_{\mathrm{g}}$ increases.

As we can notice, for the moderate values of $w_{\mathrm{g}}$ (i.e., $w_{\mathrm{g}}=1 \mathrm{~cm}$ ), similarly as in hollow-waveguide laser with classical mirrors, there exist three reflector configurations (corresponding to the dual case I, II and III) resulting in relatively high coupling efficiency. However, with decreasing Gaussian mirror (GM) parameter (the profile of the reflection coefficient of GM becomes sharper) the coupling efficiency decreases remarkably and the optimal position of the Gaussian mirror is close to the waveguide aperture only. This effect is caused by the fact that simultaneously the spot size of the reflected from GM wave decreases (for small values of $w_{\mathrm{g}}$ the spot size of the reflected wave practically equals $w_{\mathrm{g}}$ ) and, as a 
consequence, the divergence angle of the coming back wave becomes greater. This also justifies the fact that in this case the best coupling efficiency is obtained for the waveguide with greater cross-section.

Moreover, it is worth noting that the effective reflection $\rho_{\text {eff }}$ of the Gaussian mirror depends not only on $\rho_{0}$ (like in classical mirror) but also on the Gaussian mirror parameter $w_{g}$, the radius of the curvature of GM $R_{g}$, the position of the Gaussian mirror with respect to the waveguide and on the waveguide cross-section. It can be easily explained as follows. According to Eq. (2.5) $\rho_{\text {eff }}$ is not only determined by $\rho_{0}$ but also by the ratio of the spot size of incident on GM wave (which is sensitive to the cross-section of the waveguide and to the distance between the waveguide and GM) to the Gaussian mirror parameter. When this relation takes on small values the incident wave is reflected mostly by this part of the Gaussian mirror which has relatively high reflectivity coefficient. Otherwise the effective reflectivity $\rho_{\text {eff }}$ becomes small.

Thus, as we can see in Fig. 3, the effective reflectivity $\rho_{\text {eff }}$ of the Gaussian mirror decreases remarkably when we move out GM from the waveguide (the spot size of the incident wave increases) and when the Gaussian mirror parameter $w_{\mathrm{g}}$ decreases (GM has the "sharp" profile of the reflectivity coefficient).
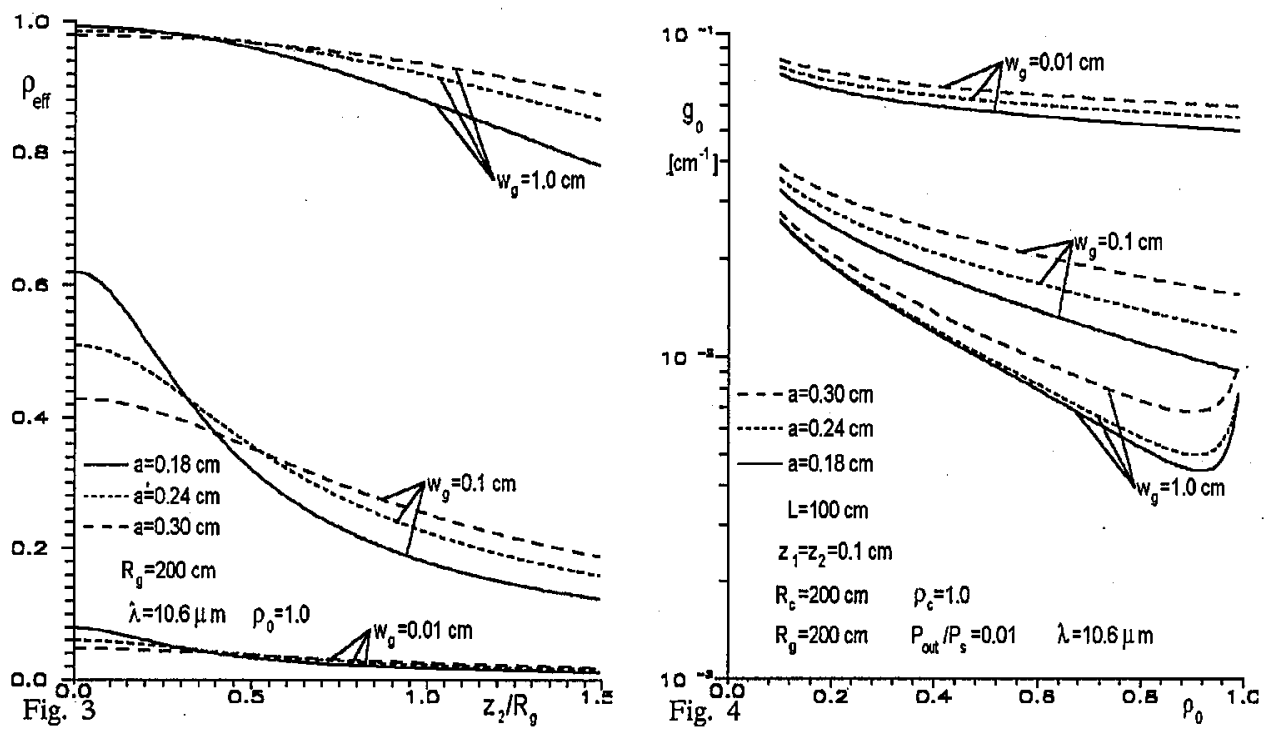

Fig. 3. Effective reflectivity of the Gaussian mirror $\rho_{\text {eff }}$ as a function of the normalised to the mirror curvature radius Gaussian mirror position $z_{2} / R_{\mathrm{g}}$ for three values of the Gaussian mirror parameter $w_{\mathrm{g}}$. Gaussian mirror peak reflectivity is equal to $\rho_{0}=1.0$. Gaussian mirror curvature radius is equal to $R_{\mathrm{g}}=200 \mathrm{~cm}$.

Fig. 4. Small signal gain $g_{0}$ as a function of the Gaussian mirror peak reflectivity $\rho_{0}$ for three values of the Gaussian mirror parameter $w_{\mathrm{g}}$ and for three values of waveguide cross-section a. Gaussian mirror peak reflectivity is equal to $\rho_{0}=1.0$. Both end mirrors curvature radii are equal to $R_{\mathrm{c}}=R_{\mathrm{g}}=200 \mathrm{~cm}$. Waveguide length is equal to $L=$ $100 \mathrm{~cm}$. Normalised output power is equal to $P_{\text {out }} / P_{\mathrm{S}}=0.01$. 
In Fig. 4 the small signal gain $g_{0}$ is plotted as a function of the output mirror peak reflectivity coefficient $\rho_{0}$ for various Gaussian mirror parameters $w_{\mathrm{g}}$ and various cross-sections of the waveguide and fixed mirror position $\left(z_{1}=z_{2}=\right.$ $0.1 \mathrm{~cm}$ ). The distributed losses outside the waveguide are neglected.

As we can notice, for small Gaussian mirror parameter (i.e., $w_{\mathrm{g}}=0.1 \mathrm{~cm}$ and $0.01 \mathrm{~cm}$ ) with increasing peak reflectivity $\rho_{0}$ the small signal gain $g_{0}$ decreases monotonically. In that case the maximal output power is obtained (for given pumping level) for $\rho_{0}=1$. However, this value of the $\rho_{0}$ does not necessarily provide maximal power efficiency of the laser system.

Furthermore, for greater values of the Gaussian mirror parameter (i.e., when GM has "soft" profile of the reflectivity coefficient) there exists optimal peak reflectivity $\rho_{0}$ resulting in small signal gain required to maintain given output power. It is worth noting that in contradiction to the hollow-waveguide laser with conventional mirrors (compare Fig. 2 in [38]) in the present case (i.e., when the cavity mirrors are close to the waveguide) the small signal gain increases with increasing waveguide cross-section. This is caused by the fact that although the volume of the active medium is enlarged but simultaneously the spot size of the wave leaving the waveguide and incident on GM is increased and, as a consequence, the effective reflectivity of Gaussian mirror becomes smaller (compare Fig. 3).

Moreover, the optimal value of $\rho_{0}$ depends on the waveguide cross-section and is shifted towards smaller values with increasing width (height) of the waveguide.

In Fig. 5 the small signal gain $g_{0}$ is plotted as a function of the normalised (to the curvature radius of the end mirrors) mirror positions for various mirror parameter $w_{\mathrm{g}}$. The height (width) of the waveguide is $a=0.18 \mathrm{~cm}$ and the output power level is $P_{\text {out }} / P_{\mathrm{S}}=0.01$. The solid lines describe the behaviour of the laser operation when the classical mirror changes its position and the Gaussian mirror is set at the waveguide aperture. The dashed lines show opposite situation.

As we can notice the laser operation depends remarkably, on which mirror is moved out from the waveguide. If we keep the Gaussian mirror close to the waveguide aperture two optimal positions (resulting in minimal values of the small signal gain) appear. In general, they correspond to dual case I and II, however they are shifted towards smaller values of the normalised distance in comparison with the positions predicted by linear analysis $[4,5,23]$. This effect is a consequence of the gain saturation in the laser and it is discussed in detail in [38].

If we change Gaussian mirror position and keep classical mirror close to the waveguide, the behaviour of the laser characteristics depends remarkably on the value of the Gaussian mirror parameter $w_{\mathrm{g}}$. For the "soft" reflection profile of the GM (i.e., $w_{\mathrm{g}}=1 \mathrm{~cm}$ ) the small signal gain behaves similarly as for the classical. mirror, and in this case also two optimal positions exist.

However, as we can notice, when the distance between end mirror and the waveguide is equal to the dual case II position (see Fig. 5) better conditions for laser operations (i.e., smaller value of the small signal gain required to obtain given level of the output power) are obtained, when the Gaussian mirror is set close to the waveguide. But when the distance between the mirror and the waveguide is greater than dual case II position, smaller values of $g_{0}$ are obtained when the 


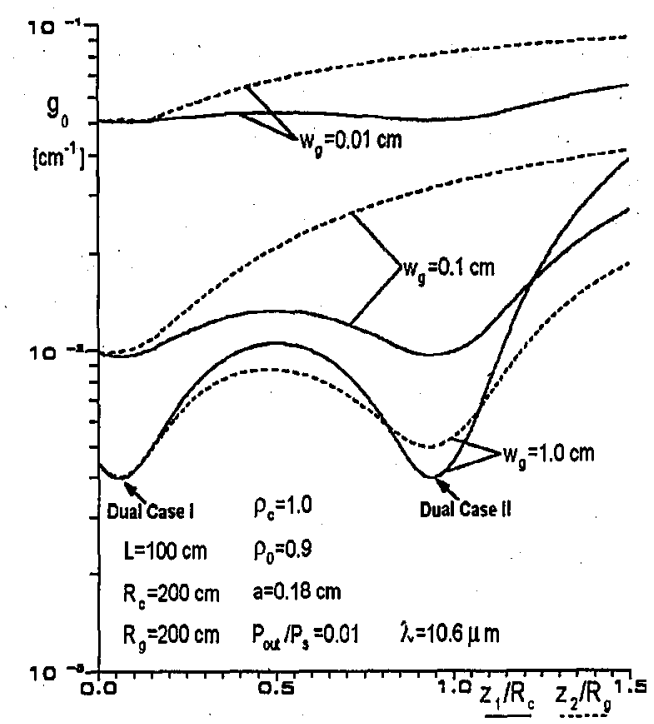

Fig. 5. Small signal gain $g_{0}$ as a function of the normalised to the mirror curvature radius classical mirror position $z_{1} / R_{\mathrm{c}}$ (solid line) and Gaussian mirror position $z_{2} / R_{\mathrm{g}}$ (dashed line) for three values of the Gaussian mirror parameter $w_{\mathrm{g}}$. Classical mirror reflectivity is equal to $\rho_{c}=1.0$. Gaussian mirror peak reflectivity is equal to $\rho_{0}=0.9$. Both end mirrors curvature radii are equal to $R_{\mathrm{c}}=R_{\mathrm{g}}=200 \mathrm{~cm}$. Waveguide length is equal to $L=100 \mathrm{~cm}$. Waveguide cross-section is equal to $a=0.18 \mathrm{~cm}$. Normalised output power is equal to $P_{\text {out }} / P_{\mathrm{S}}=0.01$.

classical mirror is at the waveguide aperture. Thus, we can say when we set an additional device (for example polarizer, $Q$-switcher, etc.) between the waveguide and the end mirror, and mirror-waveguide distance is smaller or equal to the dual case II position, we obtain a higher output power level (for given pumping level) when we put it between the classical mirror and the waveguide. In the other case, i.e. when the required distance is greater than dual case II position, the additional device should be set between GM and the waveguide (i.e. at the other side of the laser structure).

The situation is changed remarkably, when the profile of the Gaussian mirror becomes sharper. As we can notice, the small signal gain $g_{0}$ increases with the decrease in $w_{\mathrm{g}}$. This is caused by the fact that simultaneously the coupling losses and transmission losses of GM increase ( $\rho_{\text {eff }}$ decreases, see Fig. 3 ). In this case, an optimal position of the GM is only close to the waveguide. Thus the additional device should be set between classical mirror and the waveguide.

In Fig. 6 the similar characteristics are obtained for greater cross-section of the waveguide $(a=0.3 \mathrm{~cm})$. As we can notice, for the Gaussian mirror having soft profile of the reflection (i.e., $w_{\mathrm{g}}=1 \mathrm{~cm}$ ), in opposite to the previous case, there exists only one position of the Gaussian mirror as well as of the classical mirror, which corresponds to the dual case III. For greater value of $w_{\mathrm{g}}$ Gaussian mirror should be set at the waveguide aperture and classical mirror should be set at dual case III position to provide maximal output power level (for given pumping 

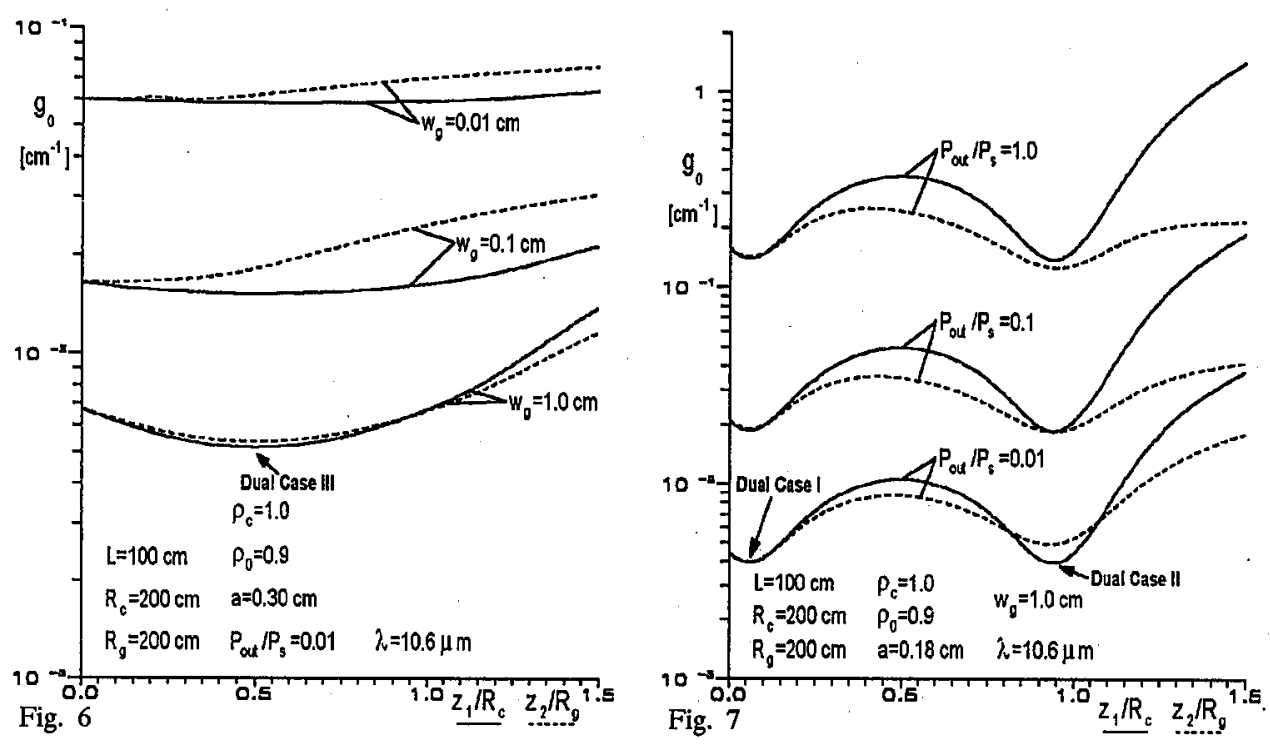

Fig. 6. Small signal gain $g_{0}$ as a function of the normalised to the mirror curvature radius classical mirror position $z_{1} / R_{\mathrm{c}}$ (solid line) and Gaussian mirror position $z_{2} / R_{\mathrm{g}}$ (dashed line) for three values of the Gaussian mirror parameter $w_{\mathrm{g}}$. Classical mirror reflectivity is equal to $\rho_{\mathrm{c}}=1.0$. Gaussian mirror peak reflectivity is equal to $\rho_{0}=0.9$. Both end mirrors curvature radii are equal to $R_{\mathrm{c}}=R_{\mathrm{g}}=200 \mathrm{~cm}$. Waveguide length is equal to $L=100 \mathrm{~cm}$. Waveguide cross-section is equal to $a=0.30 \mathrm{~cm}$. Normalised output power is equal to $P_{\text {out }} / P_{\mathrm{S}}=0.01$.

Fig. 7. Small signal gain $g_{0}$ as a function of the normalised to the mirror curvature radius classical mirror position $z_{1} / R_{\mathrm{c}}$ (solid line) and Gaussian mirror position $z_{2} / R_{\mathrm{g}}$ (dashed line) for three values of the normalised output power $P_{\text {out }} / P_{\mathrm{S}}$. Classical mirror reflectivity is equal to $\rho_{\mathrm{c}}=1.0$. Gaussian mirror peak reflectivity is equal to $\rho_{0}=0.9$. Gaussian mirror parameter is equal to $w_{\mathrm{c}}=1.0 \mathrm{~cm}$. Both end mirrors curvature radii are equal to $R_{\mathrm{c}}=R_{\mathrm{g}}=200 \mathrm{~cm}$. Waveguide length is equal to $L=100 \mathrm{~cm}$. Waveguide cross-section is equal to $a=0.18 \mathrm{~cm}$.

level). If we compare Figs. 5 and 6 we also notice that the difference between the characteristics obtained for various positions of the Gaussian and classical mirrors becomes smaller for the waveguide having greater cross-section.

In Fig. 7 the small signal gain $g_{0}$ is plotted as a function of the normalised (to the curvature mirror radius) end mirror position (solid lines correspond to the classical mirror and the dashed lines illustrated the effect of Gaussian mirror) for a normalised output power, as a parameter. The Gaussian mirror parameter is equal to $w_{\mathrm{g}}=1 \mathrm{~cm}$ and the height (width) of the waveguide is $a=0.18 \mathrm{~cm}$. In general, the laser characteristics show that the optimal position of the end mirrors corresponds to the dual case I and II configurations (because of the gain saturation effect it is slightly shifted in comparison with that predicted by linear analysis).

However, when we set an additional device between the waveguide and the Gaussian or classical mirror, an optimal position of the additional device (i.e., at 


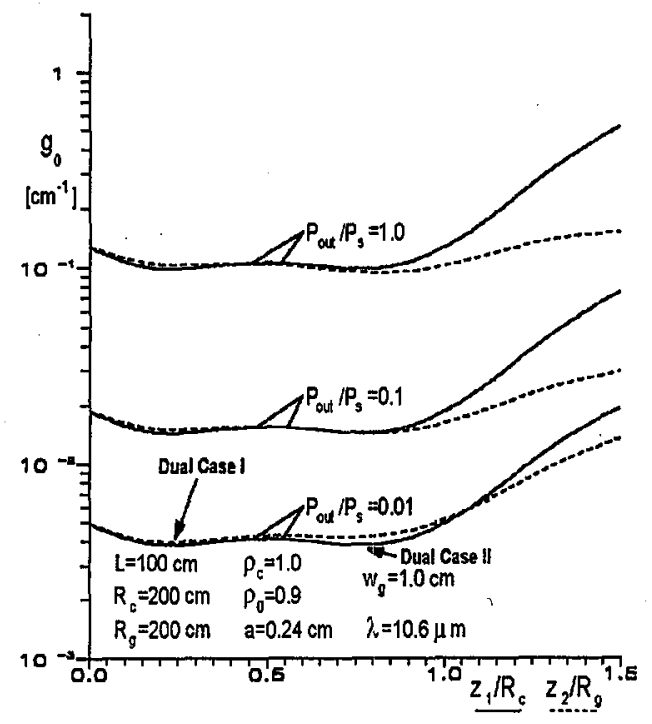

Fig. 8. Small signal gain $g_{0}$ as a function of the normalised to the mirror curvature radius classical mirror position $z_{1} / R_{\mathrm{c}}$ (solid line) and Gaussian mirror position $z_{2} / R_{\mathrm{g}}$ (dashed line) for three values of the normalised output power $P_{\text {out }} / P_{\mathrm{S}}$. Classical mirror reflectivity is equal to $\rho_{c}=1.0$. Gaussian mirror peak reflectivity is equal to $\rho_{0}=0.9$. Gaussian mirror parameter is equal to $w_{\mathrm{g}}=1.0 \mathrm{~cm}$. Both end mirrors curvature radii are equal to $R_{\mathrm{c}}=R_{\mathrm{g}}=200 \mathrm{~cm}$. Waveguide length is equal to $L=100 \mathrm{~cm}$. Waveguide cross-section is equal to $a=0.24 \mathrm{~cm}$.

which end of the waveguide it is set) depends on the output power level. As we can notice, for the higher output power levels $\left(P_{\text {out }} / P_{\mathrm{S}}>0.1\right)$ better power efficiency of the laser system is obtained, when the Gaussian mirror is moved away from the waveguide and the classical mirror is set at the aperture of the waveguide. When the waveguide has a greater cross-section, see Fig. 8 , the characteristics are less sensitive to the fact, which mirror changes its position with respect to the waveguide.

\section{Conclusions}

In this paper we have presented an approximate analysis of nonlinear operation of hollow-waveguide lasers having Gaussian mirror. Using energy approach we have derived an approximate formula that relates the small signal gain in the active medium to the output power and characteristics parameters of the laser system. The formula can be applied to study arbitrary laser configuration having end mirrors with arbitrary radii of curvature as well as Gaussian mirror parameter. Moreover, when our model is used, it is also possible to investigate the effect of extradevices introduced into the cavity on power efficiency of laser system.

It was shown that the optimal position of the Gaussian mirror corresponds to the dual case I, II and III case only for moderate and big values of the Gaussian mirror parameter (i.e., when Gaussian mirror has "soft" profile of the reflectivity 
coefficient). For sharp profile of reflection the Gaussian mirror should be set at the aperture of the waveguide but, in general, coupling losses are greater than for the classical mirror. The obtained laser characteristics showed that with the increase in Gaussian mirror parameter the higher pumping level is required to maintain the given output power level. Thus, in general, we should use the Gaussian mirror with soft profile of the reflection coefficient in order to obtain compromise between high power efficiency of the laser and high quality of the output beam.

Moreover, it was shown that for the higher output power levels, when an additional device is set into cavity, better power efficiency of the laser system is obtained when the Gaussian mirror is moved away of the waveguide. Furthermore, for increasing cross-section of the waveguide the difference between the laser characteristics obtained for different position of the classical mirror and Gaussian mirror becomes smaller.

It is also worth noting that our model can be used to study the effect of non-uniform spatial-gain distribution (depending on pumping conditions) and its influence on the optimal laser cavity configuration for maximal power efficiency of the laser system.

\section{References}

[1] E.A.J. Marcatili, R.A. Schmeltzer, The Bell System Techn. Journ. July 1964, 1783 (1964).

[2] P.W. Smith, Appl. Phys. Lett. 19, 132 (1971).

[3] H. Steffen, F.K. Kneubuhl, Phys. Lett. A 27, 612 (1968).

[4] J.J. Degnan, D.R. Hall, IEEE J. Quantum Electron. QE-9, 901 (1973).

[5] K.D. Laakmann, W.H. Steier, Appl. Opt. 15, 1334 (1976).

[6] D.M. Henderson, Appl. Opt. 15, 1066 (1976).

[7] 'C.A. Hill, D.R. Hall, IEEE J. Quantum Electron. QE-22, 1078 (1987).

[8] M. Arnz, H.E. Ponath, J. Opt. Soc. Am. B 5, 1424 (1988).

[9] C.A. Hill, IEEE J. Quantum Electron. QE-24, 1936 (1988).

[10] J. Bemnerji, A.R. Devies, P.E. Jackson, R.M. Jenkins, IEEE J. Quantum Electron. QE-26, 701 (1990).

[11] B. Schroder, IEEE J. Quantum Electron. QE-27, 158 (1991).

[12] D.G. Youmans, Appl. Phys. Lett. 44, 365 (1984).

[13] C.A. Hill, P.E. Jackson, IEEE J. Quantum Electron. QE-24, 1976 (1984).

[14] D. He, D.R. Hall, IEEE J. Quantum Electron. QE-20, 509 (1984).

[15] L.A. Newman, R.A. Hart, J.T. Kennedy, A.J. Cantor, A.J. DeMaria, Appl. Phys. Lett. 48, 1701 (1986).

[16] C.A. Hill, IEEE J. Quantum Electron. QE-23, 1968 (1987).

[17] K.H. Abramski, A.D. Colley, H.J. Baker, D.R. Hall, IEEE J. Quantum Electron. QE-27, 711 (1990).

[18] A.D. Colley, K.H. Abramski, H.J. Baker, D.R. Hall, IEEE J. Quantum Electron. QE-27, 1939 (1990).

[19] C.A. Hill, A.D. Colley, IEEE J. Quantum Electron. QE-26, 323 (1990). 
[20] J.J. Degan, Appl. Phys. 11, 1 (1976).

[21] R.L. Abrams, Laser Handbook, Vol. 3, Ed. M.L. Stitch, North-Holland, Amsterdam 1979, p. 41.

[22] P.W. Smith, O.R. Wood II, P.J. Maloney, C.R. Adams, IEEE J. Quantum Electron. QE-17, 1166 (1981).

[23] D.R. Hall, P.E. Jackson, The Physics and Technology of Laser Resonators, Hiller, Bristol 1989, Ch. 3.

[24] K. Janulewicz, P. Szczepański, Appl. Opt. 30, 3818 (1991).

[25] A. Kujawski, P. Szczepański, Opt. Eng. 31, 440 (1992).

[26] P. Szczepański, M. Skłodowska, W. Woliński, J. Lightwave Technol. 9, 329 (1991).

[27] F.T. Arecchi, E.O. Schultz, Laser Handbook, North-Holland, Amsterdam 1972.

[28] W.W. Rigrod, J. Appl. Phys. 36, 2487 (1965).

[29] W.W. Rigrod, IEEE J. Quantum Electron. QE-14, 377 (1978).

[30] G. Shindler, IEEE J. Quantum Electron. QE-15, 546 (1980).

[31] D. Eimerl, J. Appl. Phys. 51, 3008 (1980).

[32] A. Kujawski, P. Szczepański, Opt. Commun. 106, 231 (1994).

[33] T.R. Ferguson, Appl. Opt. 26, 2522 (1987).

[34] R. Ferguson, W.P. Latham, Appl. Opt. 31, 4113 (1992).

[35] D.L. Carroll, L.H. Sentman, Appl. Opt. 32, 3935 (1993).

[36] D.L. Carroll, Appl. Opt. 33, 1673 (1994).

[37] A. Kujawski, P. Szczepański, J. Mod. Opt. 38, 1901 (1991).

[38] P. Szczepański, P. Witoński, Appl. Opt. 34, 6099 (1995).

[39] G. Vakimov, Radio Eng. Electron. Phys. 10, 1439 (1965).

[40] H. Zucker, Bell Syst. Techn. J. 49, 2349 (1970).

[41] A. Piegari, G. Emiliani, Appl. Opt. 32, 5454 (1993).

[42] H. Kogelnik, T. Li, Appl. Opt. 5, 1550 (1966).

[43] N. McCarthy, P. Lavigne, Appl. Opt. 23, 3845 (1984).

[44] L.W. Casperson, IEEE J. Quantum Electron. QE-10, 629 (1974).

[45] A. Yariv, P. Yeh, Opt. Commun. 13, 370 (1975).

[46] L.W. Casperson, S.D. Lunnam, Appl. Opt. 14, 1193 (1975).

[47] U. Ganiel, A. Hardy, Appl. Opt. 9, 2145 (1976).

[48] D.M. Walsh, L.V. Knight, Appl. Opt. 25, 2947 (1986).

[49] P. Witoński, P. Szczepański, T. Adamowicz, in: Optics for Science and New Technology, Eds. J.-S. Chang, J.-H. Lee, S.-Y. Lee, Ch.H. Nam, SPIE, Vol. 2778, 1996, p. 707.

[50] A. Kujawski, P. Szczepański, J. Mod. Opt. 39, 2519 (1992). 